\title{
A correction to Hull's law on delay of reinforcement and its extension to fixed-interval operant schedules
}

\author{
ERNEST DZENDOLET \\ University of Massachusetts, Amherst, Massachusetts
}

\begin{abstract}
Hull's law on delay of reinforcement was corrected for internal consistency by testing the law against the data from which it was derived. Using these same data, the law was extended to be a function of trials. The resulting equation was extended further by mathematically transforming the latency of responding into the rate of responding, and equating the delay of reinforcement with the fixed interval of an operant schedule. The prediction of the transformed equation was that the logarithm of the rate of responding of an organism is a linear function of the logarithm of the number of trials or reinforcements, with the slope of this linear function being dependent upon the numerical value of the fixed interval.
\end{abstract}

The purpose of this paper is to show how the use of logical positivism, also termed logical empiricism, led to the formation of a new relationship from existing laws and data of learning. First, it seems appropriate to describe the basic view of logical positivism. Essentially, it is the orientation of Ernst Mach, who claimed that there is no difference between a "description" and what is generally called an "explanation" (Frank, 1949 , p. 6).

Earlier, starting in the twelfth century, a different concept of explanation had taken shape. This came via the recovery of Aristotle's logic, and of Greek and Arabic mathematics: namely, that a fact or event was explained when it could be deduced from a more general principle. In physical matters, it was obviously a physical principle (Crombie, 1959, pp. 3-8). Such a general principle could often be stated very simply. For example, the principle that "Nature abhors a vacuum," or horror vacui, was used as late as the seventeenth century in order to explain the effects produced on liquids in tubes by the newly improved air or vacuum pump (Cajori, 1962, pp. 71-77).

Later, in the thirteenth century, Grosseteste argued for the importance of mathematics in explanation. He held that it was only with mathematics that the phenomenon in question could be described accurately enough that the proper general principle would be invoked. Grosseteste's position was, therefore, that mathematics should and would describe a phenomenon, but that only physics, that is, a physical or mechanical principle, was able to say what caused the event or phenomenon. Both, however, were always required for a complete explanation (Crombie, 1959, pp. 20-22).

The break with the necessity for a duplex explanation came with the development of the philosophy of "logi-

The author's mailing address is: Department of Psychology, University of Massachusetts, Amherst, MA 01003. cal positivism" (Frank, 1949), which was later also called "logical empiricism" (Frank, 1949, pp. 45-49). In essence, this view requires that a statement about a phenomenon be made in symbols that are related to one another in a quantitative or mathematical way, so as to describe the occurrence of events in the physical world (Frank, 1949, p. 28). This view is also seen to be a current definition of a physical law, that is, a symbolic and quantitative description of a limited range of phenomena (Lindsay \& Margenau, 1957, p. 17; Planck, 1959, p. 195).

Once the mathematical statement has been properly made, that is, the law has been enunciated, mathematical manipulations of the statement may be made, leading to the prediction of facts that were not evident in the initial formulation. Finally, observations must be carried out to see whether or not the predictions made by the law are correct (Frank, 1949, pp. 18-19).

If the predictions do turn out to be correct, then the validity of the law has been supported. Furthermore, the law is now considered to have explained the phenomenon in question. There is no necessity for the other half of a duplex explanation to be brought into play. It will be remembered that this important point was brought out by Mach (Frank, 1949, p. 6), and, as mentioned earlier, that a valid law is the explanation. It must be noted, however, that not all scientists accept all these aspects of logical positivism (Planck, 1959, pp. 28-42).

\section{HULL'S LAW ON DELAY OF REINFORCEMENT}

Before proceeding to the specific law and its modification, a general view of Hull's theory can be provided by a very brief review. The term "theory" is applied . here according to the definition of Lindsay and Margenau (1957, pp. 20-21), for whom a theory is a general, mathematical relation from which a number of sub- 
sidiary laws can be derived by the use of purely mathematical operations.

Hull's theory (Hull, 1951, pp. 57-59) postulated a quantity ${ }_{S} E_{R}$, the reaction potential, which he called a quantitative theoretical construct (Hull, 1951, p. 12). It is obviously the same as an "auxiliary concept" of the logical positivists. Hull defined reaction potential as being the product of a number of other quantities, as expressed in the following equation:

$$
\mathrm{S}_{\mathrm{R}}=\mathrm{DV}_{2} \mathrm{KJ}_{\mathrm{S}} \mathrm{H}_{\mathrm{R}} \mathrm{V}_{1} \text {, }
$$

in which $\mathrm{D}$ is the drive, $\mathrm{V}_{2}$ is the stimulus-intensity dynamism at the moment of the response, $\mathrm{K}$ is the incentive reinforcement, $\mathbf{J}$ is the gradient of delay in reinforcement, $\mathrm{s}_{\mathrm{R}}$ is the habit strength, and $\mathrm{V}_{1}$ is the stimulus-intensity dynamism in the original learning. These quantities are also auxiliary concepts. It is obvious that various laws can be derived from Equation 1, depending on which parameters would be held constant and which would be allowed to vary.

To turn to the specific law, that is, the one concerning delay of reinforcement, let all parameters in Equation 1 be constant, except $J$ and ${ }_{S} H_{R}$. This leads to

$$
{ }_{S} E_{R}=A_{S} H_{R}
$$

in which $\mathrm{A}$ is a new constant that represents the combined effect of all the others. The value ${ }_{S} \mathrm{H}_{\mathrm{R}}$ is a function of trials only (Hull, 1951, pp. 29-32), and it could also become a constant if the responses of an organism were always viewed on the same trial or in the same block of trials, from one condition or experiment to another.

If Hull's theory were correct, then Equation 2 would also be correct; that is, there should be an internal consistency. Equation 2 would be easier to test if the quantities were observational concepts rather than auxiliary ones (Frank, 1949, p. 28). This conversion can be done easily, because Hull (1951, p. 56) had related J to the delay of reinforcement by

$$
\mathrm{J}=10^{-\mathrm{j} t} \text {, }
$$

in which $j$ is a constant, and had related ${ }_{S} E_{R}$ to the "reaction latency," $s_{R}{ }_{R}$, or reaction time, by

$$
S^{E_{R}}=a \cdot s^{t_{R}}{ }^{-b},
$$

in which a and b are constants (Hull, 1951,pp. 105-107). If Equations 2, 3, and 4 are combined, and ${ }_{S} H_{R}$ is considered to be a constant, as mentioned above, the result is

$$
\log _{S} t_{R}=B t+C,
$$

in which $B$ and $C$ are new constants that resulted from the algebraic manipulations. This equation now contains only observational concepts, namely, the reaction time and the delay of reinforcement. Equation 5 can be evaluated by using data reported by Perin (1943, Table 2). Because these same data were used by Hull to formulate $\mathbf{J}$ initially, the internal consistency of an aspect of Hull's theory can, therefore, be checked.

\section{CORRECTION OF HULL'S LAW}

Perin's (1943) experiment required a rat to leave a startbox, run down a short runway to a bar, and press the bar. The time between leaving the startbox and pressing the bar was the reaction time, or latency. As soon as the bar was pressed, it was withdrawn by the experimenter, and the particular delay time to be used was begun. When the delay time was completed, a food pellet was released into the food tray, the rat was allowed to eat, and then the rat was removed from the apparatus.

Figure 1 shows the logarithm of the median latency of the grouped data plotted against the delay of reinforcement, for different five-trial blocks. An exception is made in the first block, which contains only four trials. Perin (1943) listed the first trial separately, because the rat had not yet been subjected to the delay procedure. For the purpose of clarity, only 4 of the 10 trial blocks given by Perin are shown in Figure 1, and more of the earlier ones were used, because there were greater differences among these than there were among the later ones. It can be seen that none of the plots are linear, as is required by Equation 5. Therefore, Hull's theory is not internally consistent.

However, a simple transformation that improved the linearity considerably was found by trial and error. This transformation was the use of the square root of the delay of reinforcement in Equation 5. A plot of the same data, but using the new variable, is shown in Figure 2. In addition, least-squares fits were calculated

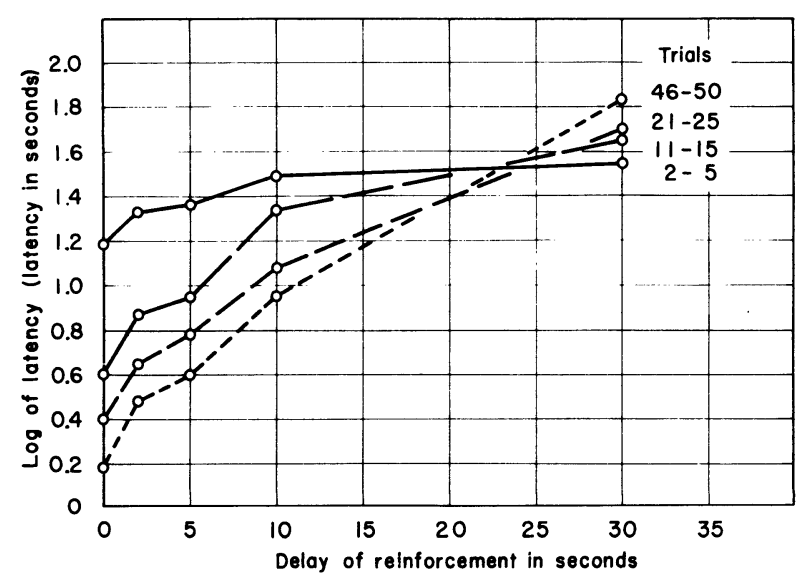

Figure 1. Median latency of responding as a function of delay of reinforcement for separate groups of rats at each delay. Data are those of Perin (1943), but not all trial blocks are shown. 


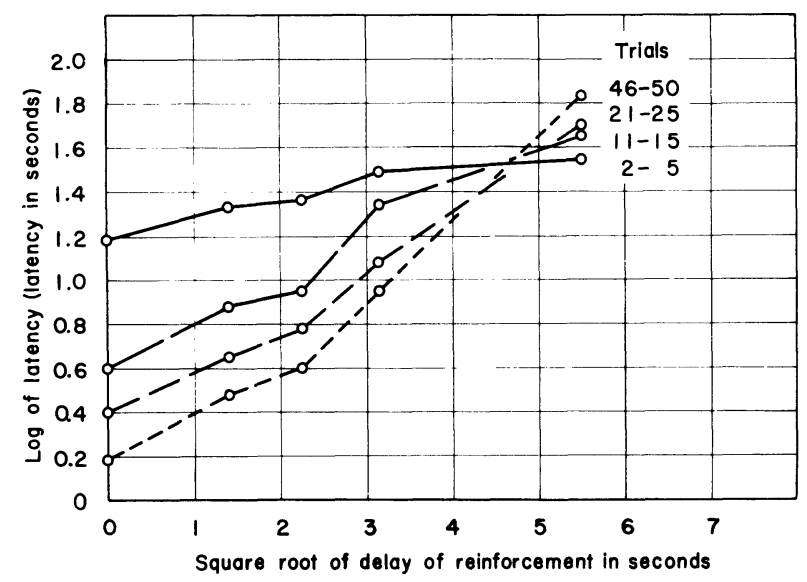

Figure 2. Same data as in Figure 1, but plotted against the square root of the delay of reinforcement. Linearity is considerably improved. Coefficients of determination for the trialblock fits are given in the text.

for all 10 trial blocks, as was the coefficient of determination, $\mathrm{r}^{2}$, for each fit. These values ranged from 0.92 to 0.98 , suggesting the existence of a good linear relationship of the data with the transformed variable. Equation 5 can then be rewritten as

$$
\log _{S} t_{R}=B \sqrt{t}+C
$$

and Hull's gradient of delay in reinforcement, $\mathrm{J}$, as

$$
J=10^{-j} \sqrt{t} .
$$

\section{EXTENSION OF THE LAW TO INCLUDE TRIALS}

To this point, Equation 6 has assumed that trials are held constant. It would be of obvious interest to extend this equation to include the effect of trials. This can be done by referring to Perin's (1943) data again. The slope of Equation 6 is B, and its value was calculated for each of the 10 trial blocks in the least-squares procedure used above. By trial and error, it was found that the slopes showed a linear, positive relationship, with $r^{2}=0.96$, when plotted against the logarithm of the trial number. Similarly, the values of the y-intercepts, $\mathrm{C}$, were calculated, and, again, a good fit was found, $r^{2}=0.97$, but a negative relation was found when the intercepts were plotted against the logarithm of the trial number. Therefore, both $\mathrm{B}$ and $\mathrm{C}$ are proportional to the logarithm of trials, with $\mathrm{B}$ being positively related and $C$ being negatively related. These relationships can be combined with Equation 6, resulting in the equation

$$
\log _{S} t_{R}=(a \sqrt{t}-b) \log T,
$$

in which $\mathrm{a}$ and $\mathrm{b}$ are proportionality constants, and $\mathrm{T}$ is trials. This equation predicts a linear relationship between the logarithm of reaction latency and the log- arithm of trials, when delay of reinforcement is held constant.

\section{APPLICATION OF THE LAW TO FIXED-INTERVAL SCHEDULES}

In applying Equation 8 to an operant conditioning situation, certain changes must be made. The first is to introduce a "rate-of-responding" variable. This can be done easily, because the latency is proportional to the reciprocal of the rate of responding. The second change, to equate the delay of reinforcement, $t$, with the duration or nominal value of the fixed interval (FI), is also easy to make. Dimensionally, there is no problem, because both have the dimension of time. There is also no problem regarding these substitutions with regard to their operational definitions. The measurement of rate of responding is generally easily carried out, as is the imposition of a desired FI value upon an organism.

The resulting new equation is

$$
\log \mathrm{R}=(\mathrm{b}-\mathrm{a} \sqrt{\mathrm{FI}}) \log \mathrm{T}+\log \mathrm{F},
$$

in which the change from Equation 8 is the replacement of $S^{t}{ }_{R}$ by $R$, the change in signs in the parentheses on the right-hand side of the equation, and the introduction of a constant of proportionality, F, because of the change from latency to rate. The equation states that the logarithm of the rate of responding of an experimental organism is a linear function of the logarithm of the trials to which the organism is subjected. The slope of this line will change as a function of the FI value in the parentheses and of the two constants $a$ and $b$, which are assumed to be characteristic of the individual organism.

According to logical empiricism, Equation 9 is a product of imagination, although based on earlier facts. If the observational concepts within it, or derived from it, are predicted to occur in a certain way, and their occurrence is subsequently observed, the equation has then explained the phenomenon in question. In spite of this view, it may be useful to discuss the initial rationale for the substitution of the duration of an FI schedule for $t$, the delay of reinforcement.

Dews (1962, 1965a, 1965b, 1966a, 1966b) reported that, in an FI schedule, the mean rate of responding in any segment within the given interval is a function of the time-position of that segment within the interval, and not of what responses were made or not made in adjacent segments or in any other positions within the interval (Dews, 1962, 1966a). This was true, regardless of the duration of the FI, which had ranged from 500 to 100,000 sec (Dews, 1965b). Dews (1966b) concluded that the typical scalloped responding was more under temporal control than under control of continuous, chained mediating behavior, and that a schedule of reinforcement should be regarded as reinforcing or determining a whole pattern of responding. 
The ability of the organism to respond according to its relative position within the FI, regardless of its duration, suggests that the organism is not responding to any absolite delay of reinforcement (Dews, 1965b); that is, the organism appears to be adjusting its responding as a function of the duration of the FI. Dews (1962) also suggested that, in any particular interval, the reference point for the organism must be the start of the interval. Because the end, that is, the presentation of the reinforcer, is also clear, only two particular times appear to be salient for the organism. These are the beginning and the end of the interval. It seems reasonable, therefore, to consider the period between these two points as the delay of reinforcement. This is also obviously the value of the FI, and can be taken as a rationale for equating the delay of reinforcement, $s_{R} t_{R}$, with the FI value in Equations 9 and 10.

At this point, some discussion is necessary with regard to the measurement of the rate of responding in an FI schedule, to decide which numerical value of the rate could be entered in Equation 9. The problem is not that of how to measure the rate, which is clearly the number of responses per unit time, but rather that of which rate to measure, because the rate is not constant throughout the interval.

It appears that the average rate over the interval would not be a good choice. Ferster and Skinner (1957, p. 142) pointed out that there may be systematic differences among the response rates within individual intervals, but that the overall rate for these various intervals would remain the same. For this reason, it seems better to use the rate within some segment within the interval. The question is which segment.

The cumulative record of responding within an FI is usually considered to follow a scalloped form, as in the articles by Dews cited earlier. However, Schneider (1969) suggested that a two-state system, in which a very sharp break occurs between the two states, may be characteristic. The first state exhibits a very low rate of responding, which abruptly changes, about twothirds of the way through the interval, into the second state, which shows a very rapid rate of responding.

Taking into account the above-mentioned characteristics, it would appear that a rate segment just before the reinforcement would be a reasonable one to choose. If the rate were constant throughout, the end segment would be equivalent to any other segment in the interval. If the responses were scalloped, the rate in the end segment would still bear a consistent relationship to the rates in all the other segments in the interval (Dews, 1966a, Figure 3 ), differing only by some constant. The end segment offers the advantage that it might be less subject than other segments to variability caused by perturbations in responding, because the end segment has the largest number of responses.
Even in the two-state system described by Schneider (1969), the end segment appears to be representative of responding in the interval. Schneider's analysis has shown that the FI value accounts for a greater proportion of the variance in the rate that is produced in the second state of the interval, that is, the faster rate, than it does in the overall rate for the interval, which he determined by including the very slow rate of the first state. He concluded that the FI value primarily influences the rate of the second state, and not that of the first state. Under these conditions, the end segment, rather than any earlier segment, is preferable, even though the rate during the second state appears to be relatively constant. The reason that it is preferable is that the second state occurs only during the last third of the interval. Use of the end segment would avoid any inadvertent inclusion of a portion of the first-state rate of responding.

\section{REFERENCES}

Cajori, F. (1962). A history of physics. New York: Dover.

Сrombie, A. C. (1959). Medieval and early modern science (Vol. 2). Garden City, NY: Doubleday.

DEws, P. B. (1962). The effect of multiple S periods on responding on a fixed-interval schedule. Journal of the Experimental Analysis of Behavior, 5, 369-374.

DEws, P. B. (1965a). The effect of multiple $S$ periods on responding on a fixed-interval schedule: II. In a primate. Journal of the Experimental Analysis of Behavior, 8, 53-54.

DEwS, P. B. (1965b). The effect of multiple $S$ periods on responding on a fixed-interval schedule: III. Effect of changes in pattern of interruptions, parameters and stimuli. Journal of the Experimental Analysis of Behavior, 8, 427-435.

Dews, P. B. (1966a). The effect of multiple $S$ periods on a fixedinterval schedule: IV. Effect of continuous $\mathrm{S}$ with only short SD probes. Journal of the Experimental Analysis of Behavior, 9, 147-151.

DEws, P. B. (1966b). The effect of multiple $S$ periods on responding on a fixed-interval schedule: V. Effect of periods of complete darkness and of occasional omissions of food presentations. Journal of the Experimental Analysis of Behavior, 9. 573-578.

Ferster, C. B., \& Skinner, B. F. (1957). Schedules of reinforcement. New York: Appleton-Century-Crofts.

Frank, P. (1949). Modern science and its philosophy. Cambridge, MA: Harvard University Press.

Hull, C. L. (1951). Essentials of behavior. New Haven, CT: Yale University Press.

Lindsay, R. B., \& Margenau, H. (1957). Foundations of physics. New York: Dover.

Perin, C. T. (1943). A quantitative investigation of the delay-ofreinforcement gradient. Journal of Experimental Psychology, 32, 37-51.

Planck, M. (1959). The new science. New York: Meridian Books.

Schneide R, B. A. (1969). A two-state analysis of fixed-interval responding in the pigeon. Journal of the Experimental Analysis of Behavior, 12, 677-687.

(Revision received for publication August 24, 1984.) 